\title{
Tracheal Tube Obstruction Due to Hemoptysis Associated With Pulmonary Infarction in a Patient With Severe COVID-19 Pneumonia
}

\author{
Takaaki Maruhashi $^{1}$, Tatsuhiko Wada ${ }^{2}$, Tomonari Masuda ${ }^{1}$, Kunihiro Yamaoka ${ }^{2}$, Yasushi Asari ${ }^{1}$ \\ 1. Department of Emergency and Critical Care Medicine, Kitasato University School of Medicine, Sagamihara, JPN 2. \\ Department of Rheumatology and Infectious Diseases, Kitasato University School of Medicine, Sagamihara, JPN
}

Corresponding author: Takaaki Maruhashi, tmaruhasi@hotmail.co.jp

\begin{abstract}
The incidence of thrombotic complications is extremely high among severe coronavirus disease 2019 (COVID-19) patients in the intensive care unit. Various factors such as a cytokine storm due to an excessive immune response to inflammation, hypoxemia, and disseminated intravascular coagulation are considered predisposing factors for thrombotic complications.
\end{abstract}

A 55-year-old Japanese man intubated eight days previously was referred to our hospital because of a severe COVID-19 pneumonia diagnosis after his pharyngeal swab tested positive for severe acute respiratory syndrome coronavirus 2 using reverse transcription-polymerase chain reaction. The patient continued to remain hypoxic $\left(\mathrm{PaO}_{2} / \mathrm{FiO}_{2}\right.$ ratio $\left.<100 \mathrm{mmHg}\right)$ at the referring hospital. On admission, we initiated venovenous extracorporeal membrane oxygenation (VV-ECMO). Unfractionated heparin and nafamostat mesylate were used as anticoagulants during VV-ECMO. Despite adequate anticoagulant therapy, he developed pulmonary infarction due to pulmonary embolism followed by hemoptysis. On day 10 following admission, his oxygen saturation dropped from $95 \%$ to $88 \%$, with a marked decrease in his ventilator tidal volume, accompanied by an inability to ventilate the patient. Thereafter, we increased the VV-ECMO flow and exchanged his endotracheal tube. The lumen of the removed tracheal tube was found to be occluded by a large-sized blood coagulum. There was no further episode of tube occlusion. The patient was discharged in a walkable state on day 39 following admission.

Endotracheal tube obstruction secondary to hemoptysis should be suggested in patients with COVID-19 requiring ventilator support, as they are unable to perform frequent endotracheal tube suctions owing to the risk of infection.

Review began $12 / 26 / 2020$ Review ended 02/21/2021 Published 02/27/2021

\section{() Copyright 2021}

Maruhashi et al. This is an open access article distributed under the terms of the Creative Commons Attribution License CC-BY 4.0., which permits unrestricted use, distribution, and reproduction in any medium, provided the original author and source are credited.
Categories: Emergency Medicine, Infectious Disease, Pulmonology

Keywords: covid-19, pulmonary embolism (pe), case report

\section{Introduction}

The coronavirus disease 2019 (COVID-19) pandemic is yet to show any sign of convergence. COVID-19 causes acute respiratory distress syndrome, and the virus may injure not only the lungs but also various other organs, such as the myocardium [1], kidney [2,3], and pancreas [4]. In addition, some recent reports have revealed the risk of embolism in severe COVID-19 cases, which is independent of the state of predisposing thromboembolic risk [5-8]. Herein, we report a rare complication of tracheal tube obstruction in a patient with severe COVID-19 pneumonia and pulmonary embolism. This article was previously posted to the Research Square preprint server on September 15, 2020.

\section{Case Presentation}

A 55-year-old Japanese man was referred to our hospital because of a severe COVID-19 pneumonia diagnosis after his pharyngeal swab tested positive for severe acute respiratory syndrome coronavirus 2 using reverse transcription-polymerase chain reaction, eight days following intubation for persistent hypoxia (PaO2/FiO2 ratio <100 mmHg). He had no history of smoking or any other underlying comorbidity, including any predisposition to thrombosis. The presence of thrombus was not confirmed at the time of admission to our hospital, even though antithrombotic therapy was not performed at the referral hospital. We initiated the patient on veno-venous extracorporeal membrane oxygenation (VV-ECMO) for providing respiratory support. During VV-ECMO, unfractionated heparin (UFH) 10000-15000 units/day and nafamostat mesylate at a fixed infusion rate of $30 \mathrm{mg} / \mathrm{h}$ were provided. Activated partial thromboplastin time (APTT) was maintained in the therapeutic range of 40-60 seconds. Despite adequate anticoagulant therapy, his Ddimer level was found to be elevated $(87.2 \mu \mathrm{g} / \mathrm{mL})$. Pulmonary embolism involving the right main pulmonary artery and right iliac vein thrombosis around the ECMO cannula insertion site in the right femoral vein was confirmed using contrast-enhanced computed tomography (CECT) (Figures 1a, 1b). CECT also identified a wedge-shaped infiltrate of poor contrast enhancement along the embolized pulmonary artery, consistent with pulmonary infarction (Figure 1c). The patient produced bloody sputum, most likely owing to pulmonary 


\section{Cureus}

infarction.
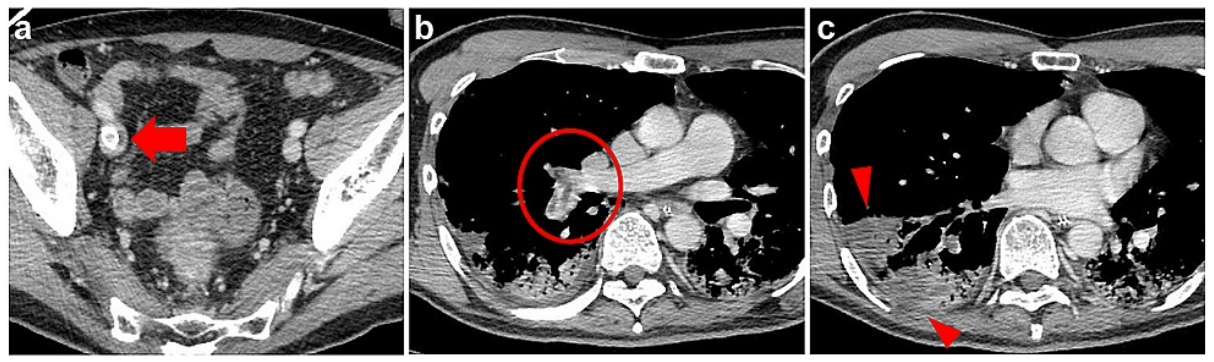

FIGURE 1: Chest-pelvis contrast-enhanced computed tomography images

(a, b) Deep vein thrombosis of the right iliac vein around the drainage cannula for extracorporeal membrane oxygenation (arrow) and pulmonary embolism of the right main pulmonary artery (circle).

(c) Pulmonary infarction, most likely secondary to a pulmonary embolism owing to the presence of poor contrast over a wedge along the embolized pulmonary artery region (arrowhead).

Nursing care, including endotracheal tube suctions during VV-ECMO, was performed at regular intervals. On day 10 following admission, the patient's oxygen saturation dropped from $95 \%$ to $88 \%$, with a marked reduction in his ventilator tidal volume, accompanied by an inability to ventilate the patient. On attempting endotracheal suctioning, the suction tube did not pass through the endotracheal tube. In response to this situation, we increased the VV-ECMO flow and performed an endotracheal tube exchange. We found that the lumen of the endotracheal tube was occluded by a large-sized blood coagulum (Figure 2).
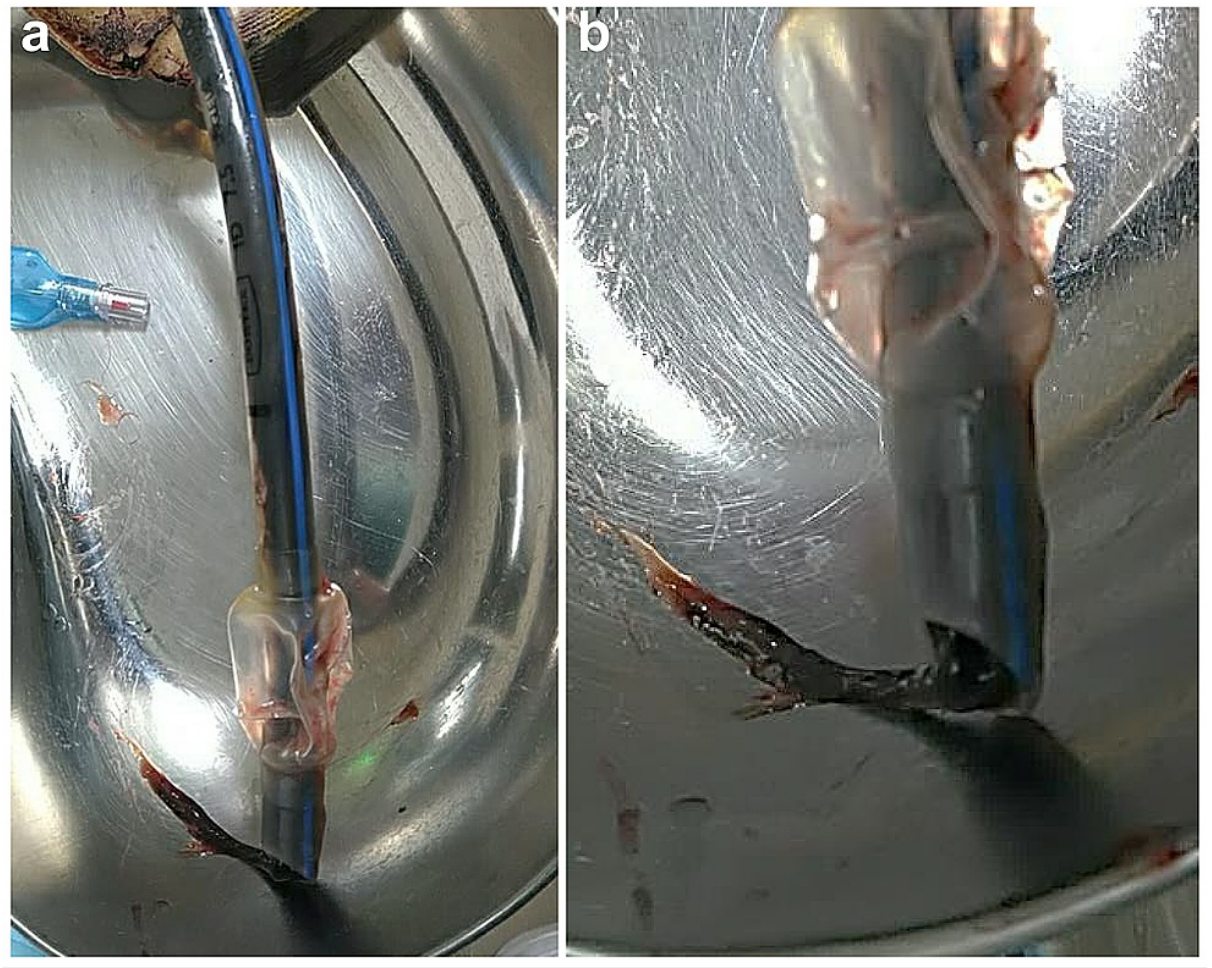

\section{FIGURE 2: Removed tracheal tube}

(a) Total length of the tracheal tube indicates the size of the coagulum.

(b) Enlarged image of the tracheal tube tip.

On day 10 , the patient's tidal volume and saturation dropped to extremely low levels. A tracheal tube exchange was performed, and ventilation failure resolved. The tube lumen was mostly occluded by blood coagulum. 
Subsequently, nafamostat mesylate was discontinued, only UFH was continued, and a tracheotomy was performed. There was no further episode of tube occlusion. The patient was taken off VV-ECMO on day 10 and discharged in a walkable state on day 39 of admission.

\section{Discussion}

The incidence of thrombotic complications among severe COVID-19 patients in intensive care units (ICU) is extremely high (31\%) [6]. The predisposing factors for thrombotic complications in COVID-19 patients include various factors such as a cytokine storm due to an excessive immune response to inflammation, hypoxemia, and disseminated intravascular coagulation [9-12].

In our case, the patient was initiated on VV-ECMO and received UFH. The UFH dose was titrated carefully based on APTT monitoring. Our patient developed pulmonary embolism despite these measures. A pulmonary embolism leads to pulmonary infarction, as in this case, and among patients with pulmonary infarction, 13\% develop hemoptysis [13]. The complication of endotracheal tube block by blood coagulum may be explained by the coexistence of hypercoagulability and bleeding tendency against the background of COVID-19 pneumonia. Therefore, severe COVID-19 patients may require careful monitoring of blood coagulation status and devising antithrombotic therapy.

We also considered other possible causes of endotracheal tube obstruction. The risk of health care workers (HCWs) acquiring an infection, as they are involved in the care of patients with COVID-19, is approximately 1.1\%. This risk may increase during tracheal tube insertion and endotracheal suctioning, procedures known to generate aerosols [14]. Consequently, it is possible that frequent endotracheal tube suctioning, as is normally recommended in the ICU management of intubated patients with severe COVID-19 pneumonia, may not have been performed in this case. Measures to minimize the risk of an HCW acquiring an infection while performing endotracheal suctioning are warranted as well as increasing their confidence in adhering to the required protocols. This may reduce the incidence of endotracheal tube blockage among patients managed on ventilators. Fortunately, in our case, we could exchange the obstructed tracheal tube because of the respiratory assistance provided by VV-ECMO. However, in the absence of VV-ECMO, urgent measures are required for a rapid exchange of endotracheal tube.

\section{Conclusions}

In patients with severe COVID-19 requiring ventilator support, a high suspicion of endotracheal tube obstruction secondary to various factors should be maintained to avoid adverse outcomes.

\section{Additional Information \\ Disclosures}

Human subjects: Consent was obtained or waived by all participants in this study. Conflicts of interest: In compliance with the ICMJE uniform disclosure form, all authors declare the following: Payment/services info: All authors have declared that no financial support was received from any organization for the submitted work. Financial relationships: All authors have declared that they have no financial relationships at present or within the previous three years with any organizations that might have an interest in the submitted work. Other relationships: All authors have declared that there are no other relationships or activities that could appear to have influenced the submitted work.

\section{References}

1. Chen L, Li X, Chen M, Feng Y, Xiong C: The ACE2 expression in human heart indicates new potential mechanism of heart injury among patients infected with SARS-CoV-2. Cardiovasc Res. 2020, 116:10971100. $10.1093 / \mathrm{cvr} / \mathrm{cvaa} 078$

2. Hirsch JS, Ng JH, Ross DW, et al.: Acute kidney injury in patients hospitalized with COVID-19 . Kidney Int. 2020, 98:209-218. 10.1016/j.kint.2020.05.006

3. Martínez-Rojas MA, Vega-Vega O, Bobadilla NA: Is the kidney a target of SARS-CoV-2? . Am J Physiol Renal Physiol. 2020, 318:F1454-F1462. 10.1152/ajprenal.00160.2020

4. Mukherjee R, Smith A, Sutton R: Covid-19-related pancreatic injury. Br J Surg. 2020, 107:e190. 10.1002/bjs. 11645

5. Bikdeli B, Madhavan MV, Jimenez D, et al.: COVID-19 and thrombotic or thromboembolic disease: implications for prevention, antithrombotic therapy, and follow-up. J Am Coll Cardiol. 2020, 75:2950-2973. 10.1016/j.jacc.2020.04.031

6. Klok FA, Kruip MJ, van der Meer NJ, et al.: Incidence of thrombotic complications in critically ill ICU patients with COVID-19. Thromb Res. 2020, 191:145-147. 10.1016/j.thromres.2020.04.013

7. Chen N, Zhou M, Dong X, et al.: Epidemiological and clinical characteristics of 99 cases of 2019 novel coronavirus pneumonia in Wuhan, China: a descriptive study. Lancet. 2020, 395:507-513. 10.1016/S01406736(20)30211-7

8. Tang N, Li D, Wang X, Sun Z: Abnormal coagulation parameters are associated with poor prognosis in patients with novel coronavirus pneumonia. J Thromb Haemost. 2020, 18:844-847. 10.1111/jth.14768

9. Chen T, Wu D, Chen H, et al.: Clinical characteristics of 113 deceased patients with coronavirus disease 2019: retrospective study. BMJ. 2020, 368:m1091. 10.1136/bmj.m1091 


\section{Cureus}

10. Guan WJ, Ni ZY, Hu Y, et al.: Clinical characteristics of coronavirus disease 2019 in China . N Engl J Med. 2020, 382:1708-1720. 10.1056/NEJMoa2002032

11. Wang D, Hu B, Hu C, et al.: Clinical characteristics of 138 hospitalized patients with 2019 novel coronavirus-infected pneumonia in Wuhan, China. JAMA. 2020, 323:1061-1069. 10.1001/jama.2020.1585

12. Zhou F, Yu T, Du R, et al.: Clinical course and risk factors for mortality of adult inpatients with COVID-19 in Wuhan, China: a retrospective cohort study. Lancet. 2020, 395:1054-1062. 10.1016/S01406736(20)30566-3

13. Casey K, Iteen A, Nicolini R, Auten J: COVID-19 pneumonia with hemoptysis: Acute segmental pulmonary emboli associated with novel coronavirus infection. Am J Emerg Med. 2020, 38:1544.e1-1544.e3.

10.1016/j.ajem.2020.04.011

14. Lai X, Wang M, Qin C, et al.: Coronavirus disease 2019 (COVID-2019) infection among health care workers and implications for prevention measures in a tertiary hospital in Wuhan, China. JAMA Netw Open. 2020, 3:e209666. 10.1001/jamanetworkopen.2020.9666 